\title{
Mapping QTL for growth and muscling traits in three connected porcine $\mathrm{F}_{2}$ crosses
}

\author{
Christine Rückert, Patrick Stratz, Siegfried Preuss and Jörn Bennewitz
}

Institute of Animal Husbandry and Animal Breeding, Universität Hohenheim, Stuttgart-Hohenheim, Germany

\begin{abstract}
QTL experiments in pigs are often analysed separately, although similar or same founder breeds are frequently used to establish the experimental design. The aim of the present study was to jointly analyse three porcine $F_{2}$-crosses for six growth and four muscling traits. The crosses were a Meishan $\times$ Pietrain cross, a Wild Boar $\times$ Pietrain cross, and a Wild Boar $\times$ Meishan cross. In some cases, same founder animals were used to establish the crosses. $966 \mathrm{~F}_{2}$-individuals were genotyped for 242 genetic markers (mostly microsatellites) and phenotyped for birth weight, 21 and 35 day weight, slaughter weight, carcass length, food conversion ratio, ham meat weight, shoulder meat weight, loin and neck meat weight, and meat area. A multi-allele multi-QTL model was applied that estimated an additive QTL effect for each founder breed and parental origin (either paternally or maternally derived), and a dominant QTL effect for each cross. This model was previously introduced in plant breeding. Numerous QTL were mapped on the autosomes. Most QTL were localised on SSC1, 2, 3, 4, 6 and 8, and no QTL were on SSC9, 11, 13, 15, 17 and 18. The confidence intervals were short in many cases. QTL with an exceptionally high test statistic were found for carcass length on $\mathrm{SSC} 1,4,7$ and 17. The coefficient of variation was remarkably small for this trait, which suggests that carcass length is affected by only a few genes with large effects. Positional and functional candidates underlying promising QTL are suggested for further study.
\end{abstract}

Keywords: joint analysis, QTL, growth and muscling traits

\section{Introduction}

QTL mapping has received considerable attention in animal breeding over the last two decades. Experimental designs can be classified into two groups: those using existing family structure, e.g. half-sib families, or those based on experimental crosses. For mapping QTL on the pig genome, $F_{2}$-experimental crosses were often established from two founder breeds (Andersson et al. 1994, Rothschild et al. 2007). Although numerous $F_{2}$-designs with same founder breeds exist, they were usually analysed separately, probably because they were established by different research groups. However, it has frequently been shown that a combined analysis of QTL experiments boosts the statistical power substantially (Walling et al. 2000, Bennewitz et al. 2003). The three $\mathrm{F}_{2}$-designs established by Geldermann et al. (2003) are especially well suited for a joint analysis, because not only same founder breeds, but also same founder animals were used to set up the designs. 
Rückert \& Bennewitz (2010) proposed a model adapted from plant breeding for analysis of connected $\mathrm{F}_{2}$-experiments and showed the benefit of a joint analysis of these three designs. It was shown that the model not only increased the statistical power in a joint analysis, but also the confidence intervals of QTL positions were remarkably small given that only linkage information was used. This model was successfully applied to map QTL for metabolic and cytological fat traits by Rückert et al. (2012). Stratz et al. (2012) mapped QTL for meat quality traits considering main and pairwise epistatic effects. The aim of the present study was to map QTL for growth and muscling traits in the three $F_{2}$-designs from Geldermann et al. (2003) using the approach of Rückert \& Bennewitz (2010).

\section{Material and methods}

The experimental design consisted of a Meishan $(M) \times$ Pietrain $(P) F_{2}$ cross $(M \times P)$, a European Wild Boar $(\mathrm{W}) \times \mathrm{PF}_{2}$ cross $(\mathrm{W} \times \mathrm{P})$, and a $\mathrm{W} \times \mathrm{MF}_{2}$ cross. The number of individuals in each cross and generation can be found in Table 1. Some founder animals were the same in different crosses, e.g. the same $\mathrm{W}$ boar was used to generate the $\mathrm{W} \times \mathrm{P}$ and the $\mathrm{W} \times \mathrm{M}$ cross. A detailed description of the design can be found in Geldermann et al. (2003). The $F_{2}$-individuals were phenotyped for numerous traits. In this study, growth traits (birth weight, 21 day weight, 35 day weight, live weight at slaughter, food conversion ratio, and carcass length) and muscling traits (ham meat weight, shoulder meat weight, loin and neck meat weight and meat area between the $13 \mathrm{th} / 14$ th rib in the $m$. longissimus dorsi) were analysed, see Table 2.

Table 1

Overview of the three crosses generated by mating Meishan (M) with Pietrain (P), Wild Boar (W) with P and W with M

\begin{tabular}{|c|c|c|c|c|c|c|c|}
\hline Cross & M & $\times$ & W & $\times$ & W & M & $\Sigma$ \\
\hline Sex & $0^{\prime \prime}$ & o & $0^{\prime \prime}$ & o & $0^{x}$ & 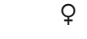 & \\
\hline No. of founder animals & 1 & 8 & 1 & 9 & 1 & 4 & 24 \\
\hline No. of animal in the $F_{1}$ & 3 & 19 & 2 & 26 & 2 & 21 & 73 \\
\hline No. of animal in the $F_{2}$ & 170 & 146 & 150 & 165 & 169 & 166 & 966 \\
\hline
\end{tabular}

Table 2

Traits and the abbreviations used in this paper

\begin{tabular}{|c|c|c|c|}
\hline Group & Trait & Abbr. & Symbols used in Figure 1 \\
\hline \multirow[t]{6}{*}{ Growth } & Birthweight & BW & $\nabla$ \\
\hline & 21 day weight & W21 & $\triangle$ \\
\hline & 35 day weight & W35 & + \\
\hline & Live weight at slaughter & SW & $\bigcirc$ \\
\hline & Food conversion ratio & FCR & $x$ \\
\hline & Carcass length & $\mathrm{CL}$ & $\square$ \\
\hline \multirow[t]{4}{*}{ Muscling } & Ham meat weight & HMW & $\diamond$ \\
\hline & Sholder meat weight & SMW & $\mathbf{\square}$ \\
\hline & Loin and neck meat weight & LNMW & $\bullet$ \\
\hline & Meat area between 13th/14th rib in m. longissimus dorsi & MA & $\Delta$ \\
\hline
\end{tabular}

Data recording took place under standardised conditions at one experimental farm. The means and standard deviations of the traits in the crosses are shown in Table 3. The data were pre-corrected for the effect of the litter, the sex and age at slaughter. 
All animals were genotyped for 242 genetic markers. These marker data were linked to the pedigree and a common genetic map was calculated and presented by Rückert \& Bennewitz (2010). Because many markers were genotyped in two or three crosses this calculation was straightforward. QTL analysis was done using the multi-allele multi-QTL model of Rückert \& Bennewitz (2010). The model assumes that two founder breeds $i$ and $j$ of an $F_{2}$ individual are divergent homozygous at a putative QTL. Under this assumption, for each $\mathrm{F}_{2}$ individual and each chromosomal position (i.e. each cM) the following four genotype probabilities were estimated, $p r$ $\left(Q_{i}^{p} Q_{i}^{m}\right), \operatorname{pr}\left(Q_{j}^{p} Q_{i}^{m}\right) \operatorname{pr}\left(Q_{i}^{p} Q_{j}^{m}\right)$ and $\operatorname{pr}\left(Q_{j}^{p} Q_{j}^{m}\right)$, using a modified version of BigMap (Reinsch 1999).

Table 3

Number of observations (n), mean, standard deviation (SD), minimum (Min) and maximum (Max) of the phenotypic observations and coefficient of variation (CV)

\begin{tabular}{|c|c|c|c|c|c|c|c|}
\hline Trait & Cross & $n$ & Mean & SD & Min & Max & CV \\
\hline BW & $M \times P$ & 316 & 14.01 & 3.15 & 5.00 & 23.00 & 22.49 \\
\hline \multirow[t]{3}{*}[\mathrm{kg}^{*}10]{} & $W \times P$ & 315 & 14.06 & 2.99 & 5.00 & 26.00 & 21.30 \\
\hline & $W \times M$ & 335 & 12.60 & 2.04 & 7.00 & 20.00 & 16.19 \\
\hline & Joint & 966 & 13.54 & 2.84 & 5.00 & 26.00 & 20.97 \\
\hline W21 & $M \times P$ & 303 & 60.22 & 11.02 & 16.00 & 90.00 & 18.30 \\
\hline \multirow[t]{3}{*}[\mathrm{kg}^{*}10]{} & $W \times P$ & 315 & 45.49 & 12.01 & 14.00 & 81.00 & 26.40 \\
\hline & $W \times M$ & 334 & 46.64 & 11.12 & 17.00 & 80.00 & 23.84 \\
\hline & Joint & 952 & 50.58 & 13.16 & 14.00 & 90.00 & 26.02 \\
\hline W35 & $M \times P$ & 316 & 88.60 & 15.66 & 39.00 & 135.00 & 17.67 \\
\hline \multirow[t]{3}{*}[\mathrm{kg}^{*}10]{} & $W \times P$ & 315 & 68.67 & 16.29 & 28.00 & 116.00 & 23.72 \\
\hline & $W \times M$ & 329 & 64.95 & 17.97 & 21.00 & 115.00 & 27.66 \\
\hline & Joint & 960 & 73.96 & 19.63 & 21.00 & 135.00 & 26.55 \\
\hline SW & $M \times P$ & 316 & 96.07 & 16.84 & 27.00 & 139.00 & 17.53 \\
\hline \multirow[t]{3}{*}[\mathrm{kg}]{} & $\mathrm{W} \times \mathrm{P}$ & 314 & 72.37 & 14.62 & 28.00 & 108.00 & 20.20 \\
\hline & $W \times M$ & 335 & 71.16 & 13.79 & 23.00 & 107.00 & 19.38 \\
\hline & Joint & 965 & 79.71 & 18.94 & 23.00 & 139.00 & 23.76 \\
\hline $\mathrm{CL}$ & $M \times P$ & 316 & 91.33 & 6.08 & 63.50 & 106.00 & 6.66 \\
\hline \multirow[t]{3}{*}[\mathrm{cm}]{} & $W \times P$ & 315 & 79.89 & 5.19 & 62.50 & 94.00 & 6.50 \\
\hline & $\mathrm{W} \times \mathrm{M}$ & 335 & 78.21 & 5.40 & 56.00 & 92.50 & 6.90 \\
\hline & Joint & 966 & 83.05 & 8.05 & 56.00 & 106.00 & 9.69 \\
\hline FCR & $M \times P$ & 316 & 3.88 & 0.88 & 2.60 & 11.46 & 22.59 \\
\hline \multirow[t]{3}{*}[\mathrm{kg}/\mathrm{kg}]{} & $W \times P$ & 315 & 3.42 & 0.50 & 2.54 & 8.83 & 14.66 \\
\hline & $W \times M$ & 335 & 4.32 & 0.68 & 2.81 & 7.03 & 15.64 \\
\hline & Joint & 966 & 3.88 & 0.79 & 2.54 & 11.46 & 20.38 \\
\hline HMW & $M \times P$ & 316 & 7.09 & 1.26 & 2.00 & 11.20 & 17.78 \\
\hline \multirow[t]{3}{*}[\mathrm{kg}]{} & $W \times P$ & 315 & 6.58 & 1.33 & 2.60 & 10.70 & 20.25 \\
\hline & $W \times M$ & 335 & 4.44 & 0.76 & 1.55 & 6.35 & 17.08 \\
\hline & Joint & 966 & 6.00 & 1.62 & 1.55 & 11.20 & 27.02 \\
\hline SMW & $M \times P$ & 316 & 3.64 & 0.63 & 1.15 & 5.65 & 17.25 \\
\hline \multirow[t]{3}{*}[\mathrm{kg}]{} & $W \times P$ & 315 & 3.27 & 0.67 & 1.30 & 5.35 & 20.51 \\
\hline & $\mathrm{W} \times \mathrm{M}$ & 335 & 2.41 & 0.45 & 1.00 & 3.90 & 18.53 \\
\hline & Joint & 966 & 3.09 & 0.78 & 1.00 & 5.65 & 25.34 \\
\hline LNMW & $M \times P$ & 316 & 6.48 & 1.17 & 1.70 & 10.10 & 18.11 \\
\hline \multirow[t]{3}{*}{ [kg] } & $W \times P$ & 315 & 5.55 & 1.26 & 1.95 & 10.05 & 22.65 \\
\hline & $W \times M$ & 335 & 3.82 & 0.70 & 1.30 & 6.05 & 18.32 \\
\hline & Joint & 966 & 5.25 & 1.54 & 1.30 & 10.10 & 29.28 \\
\hline MA & $M \times P$ & 316 & 29.29 & 5.35 & 14.56 & 49.31 & 18.26 \\
\hline \multirow[t]{3}{*}[\mathrm{cm}^{*}\mathrm{cm}]{} & $\mathrm{W} \times \mathrm{P}$ & 313 & 32.71 & 6.40 & 12.93 & 50.05 & 19.57 \\
\hline & $\mathrm{W} \times \mathrm{M}$ & 335 & 19.42 & 3.13 & 7.73 & 31.81 & 16.13 \\
\hline & Joint & 964 & 26.97 & 7.64 & 7.73 & 50.05 & 28.32 \\
\hline
\end{tabular}


The upper subscripts denote the parental origin of the alleles (i.e. paternally $(p)$ or maternally $(m)$ derived) and the lower subscripts denote the breed origin of the alleles (i.e. breed $i$ or $j$, with $i, j$ being breed $\mathrm{M}, \mathrm{P}$, or $\mathrm{W}$ ). These probabilities were used in a regression framework to estimate an additive QTL effect for each founder breed and each parental origin, i.e. $\hat{a}_{M^{\prime}}^{p} \hat{a}_{M^{\prime}}^{m} \hat{a}_{p^{\prime}}^{p}$ $\hat{a}_{p^{\prime}}^{m} \hat{a}_{w^{\prime}}^{p} \hat{a}_{w^{\prime}}^{m}$ where the lower subscript denotes the breed and the upper subscript denotes the parental origin. Additionally, a dominant QTL effect was estimated for each cross. The effect of the crosses was included and the residual variance was modelled to be heterogeneous across the crosses. The model was fitted for each $\mathrm{cM}$ on the autosomes. The test statistic was an F-test. The null hypothesis was that every estimate (i.e. each additive and dominant QTL effect estimated) at the position with the highest test statistic on a chromosome was equal to zero. The alternative hypothesis was that at least one effect was different from zero at this position. Correction for multiple testing on a chromosome was done using the quick method of Piepho (2001), accepting a $5 \%$ error probability for significance. This somewhat loose threshold value was chosen because it was shown that many QTL with small effects segregate in these crosses (Bennewitz \& Meuwissen 2010). At significant chromosomal positions it was tested if the additive and / or the imprinting and / or the dominant QTL effect were significant. These tests were conducted by building linear contrasts and resulted in the three error probabilities $p_{\text {add }} p_{\text {dom }}$ and $p_{\text {imp }}$ for additive, dominance and imprinting QTL, respectively. Additionally, the number of QTL alleles was determined based on their mendelian effects (i.e. ignoring parental origin of the alleles, $\hat{a}_{p^{\prime}} \hat{a}_{M^{\prime}} \hat{a}_{w^{\prime}}$ ). QTL confidence intervals were obtained by the one LOD drop method (Lynch \& Walsh 1998). For this purpose, F-values were converted into LOD-scores. Multiple QTL were included as cofactors in the model using a forward selection approach. This increased statistical power and enabled the detection of multiple QTL on a chromosome. A more detailed description of this procedure can be found in Rückert \& Bennewitz (2010).

\section{Results and discussion}

The summary statistics in Table 1 reveal substantial variation for all traits within and across the three crosses. However, a low coefficient of variation was observed for CL. For the growth traits W21, W35 and CL, and SW the mean of the M $\times P$ cross is substantially higher than the mean of the other two crosses. For BW, HMW, and MA the $\mathrm{W} \times \mathrm{M}$ cross mean is substantially lower. This is in agreement with the history of the breeds. The Pietrain breed is a typical sire line used to generate crosses for slaughter pigs, and was selected for growth and meat quality during the last decades. The Meishan breed is known to be a fatty and fertile breed. Wild Boar is a small size breed. It was not subject to artificial selection and hence little or no selection pressure was on growth traits. The QTL results for growth traits and muscling traits are shown in Table 4 and 5, respectively. For many QTL with significant additive effects three Mendelian alleles could be observed. In this case, the order of effects was often, but not always, $\hat{a}_{p}>\hat{a}_{M}>\hat{a}_{w}$. If only two Mendelian alleles were observed, the order of effects was often $\hat{a}_{p}=\hat{a}_{M}>\hat{a}_{w^{\prime}}$ or $\hat{a}_{p}>\hat{a}_{M}=\hat{a}_{w}$. This was expected due to the selection history of the breeds mentioned above, but it also indicates genetic variation for these traits within the founder breeds. 
Table 4

QTL results for growth traits

\begin{tabular}{|c|c|c|c|c|c|c|c|c|c|c|}
\hline \multirow{2}{*}{$\frac{\text { Trait }}{\text { BW }}$} & \multirow{2}{*}{$\frac{\text { SSC }}{8}$} & \multirow{2}{*}{$\frac{\text { Pos }}{6}$} & \multicolumn{2}{|l|}{$\mathrm{Cl}$} & \multirow{2}{*}{$\frac{\text { F-value }}{3.97}$} & \multirow{2}{*}{$\frac{P_{\text {add }}}{0.0005}$} & \multirow{2}{*}{$\frac{P_{\text {dom }}}{0.0172}$} & \multirow{2}{*}{$\frac{P_{\text {imp }}}{0.1980}$} & \multirow{2}{*}{$\frac{\text { Mode }}{(--)}$} & \multirow{2}{*}{$\frac{\text { Order of effects }}{\hat{a}_{\mathrm{p}}>\hat{a}_{\mathrm{M}}>\hat{a}_{w}}$} \\
\hline & & & $\begin{array}{l}{[0.0 ;} \\
\text { [SW905; }\end{array}$ & $\begin{array}{r}18.0] \\
\text { SW933] }\end{array}$ & & & & & & \\
\hline \multirow[t]{4}{*}{ W21 } & 6 & 101 & $\begin{array}{l}{[96.4 ;} \\
\text { [RYR; }\end{array}$ & $\begin{array}{r}106.0] \\
\text { SKI] }\end{array}$ & 4.62 & 0.0017 & 0.0050 & 0.1273 & $(--)$ & $\hat{a}_{\mathrm{m}}>\hat{a}_{\mathrm{p}}=\hat{a}_{\mathrm{w}}$ \\
\hline & 8 & 3 & $\begin{array}{l}{[0.0 ;} \\
{[S W 905 ;}\end{array}$ & $\begin{array}{r}18.0] \\
\text { SW933] }\end{array}$ & 3.99 & $<0.0001$ & 0.1194 & 0.6099 & $(--)$ & $\hat{a}_{\mathrm{p}}>\hat{a}_{\mathrm{M}}>\hat{a}_{\mathrm{w}}$ \\
\hline & 15 & 99 & $\begin{array}{l}\text { [71.9; } \\
\text { [SW2053; }\end{array}$ & $\begin{array}{r}99.4] \\
\text { SW1983] }\end{array}$ & 3.27 & 0.6207 & 0.0150 & 0.0030 & (nc) & $\hat{a}_{\mathrm{m}}=\hat{a}_{\mathrm{p}}=\hat{a}_{\mathrm{w}}$ \\
\hline & 16 & 10 & $\begin{array}{l}{[0.0 ;} \\
{[S 0111 ;}\end{array}$ & $\begin{array}{r}33.3] \\
\text { SW419] }\end{array}$ & 3.24 & 0.0327 & 0.0363 & 0.0139 & (nc) & $\hat{a}_{w}>\hat{a}_{M}=\hat{a}_{p}$ \\
\hline \multirow[t]{5}{*}{ W35 } & 6 & 100 & $\begin{array}{l}{[96.4 ;} \\
\text { [RYR; }\end{array}$ & $\begin{array}{r}106.0] \\
\text { SKI] }\end{array}$ & 4.21 & 0.0014 & 0.0152 & 0.1513 & $(--)$ & $\hat{a}_{\mathrm{M}}>\hat{\mathrm{a}}_{\mathrm{p}}=\hat{\mathrm{a}}_{\mathrm{w}}$ \\
\hline & 8 & 5 & $\begin{array}{l}{[0.0 ;} \\
\text { [SW905; }\end{array}$ & $\begin{array}{r}34.0] \\
\text { SW933] }\end{array}$ & 3.82 & $<0.0001$ & 0.0393 & 0.8651 & $(--)$ & $\hat{a}_{p}>\hat{a}_{M}=\hat{a}_{w}$ \\
\hline & 12 & 1 & $\begin{array}{l}{[0.0 ;} \\
\text { [S0143; }\end{array}$ & $\begin{array}{l}10.8] \\
\text { EAD] }\end{array}$ & 3.22 & 0.7210 & 0.0748 & 0.0006 & (mat) & $\hat{a}_{\mathrm{m}}=\hat{a}_{\mathrm{p}}=\hat{a}_{\mathrm{w}}$ \\
\hline & 12 & 75 & $\begin{array}{l}\text { [64.5; } \\
\text { [SW874; }\end{array}$ & $\begin{array}{r}99.3] \\
\text { S0174] }\end{array}$ & 3.45 & 0.2497 & 0.0577 & 0.0016 & (nc) & $\hat{a}_{\mathrm{m}}=\hat{a}_{\mathrm{p}}>\hat{a}_{\mathrm{w}}$ \\
\hline & 14 & 132 & $\begin{array}{l}\text { [105.1; } \\
\text { [SW2488; }\end{array}$ & $\begin{array}{r}151.3] \\
\text { SW2515] }\end{array}$ & 2.60 & 0.0081 & 0.0544 & 0.9052 & $(--)$ & $\hat{a}_{p}>\hat{a}_{M}=\hat{a}_{w}$ \\
\hline \multirow[t]{8}{*}{ SW } & 1 & 90 & $\begin{array}{l}{[77.3 ;} \\
\text { [SW2130; }\end{array}$ & $\begin{array}{r}\text { 104.1] } \\
\text { IGFR] }\end{array}$ & 7.99 & $<0.0001$ & 0.9368 & 0.0118 & (nc) & $\hat{a}_{\mathrm{M}}=\hat{a}_{\mathrm{p}}>\hat{a}_{\mathrm{w}}$ \\
\hline & 2 & 76 & $\begin{array}{l}\text { [70.6; } \\
\text { [MYOD1; }\end{array}$ & $\begin{array}{r}\text { 78.3] } \\
\text { INSR] }\end{array}$ & 4.84 & $<0.0001$ & 0.0095 & 0.3624 & $(--)$ & $\hat{a}_{m}>\hat{a}_{p}>\hat{a}_{w}$ \\
\hline & 3 & 59 & $\begin{array}{l}{[50.8 ;} \\
{[O I F ;}\end{array}$ & $\begin{array}{r}74.0] \\
\text { SW828] }\end{array}$ & 3.18 & 0.0205 & 0.0038 & 0.6224 & $(--)$ & $\hat{a}_{\mathrm{M}}=\hat{a}_{\mathrm{p}}>\hat{a}_{\mathrm{w}}$ \\
\hline & 4 & 71 & $\begin{array}{l}\text { [62.1; } \\
\text { [SW1073; }\end{array}$ & $\begin{array}{r}75.3] \\
\text { S0073] }\end{array}$ & 5.62 & $<0.0001$ & 0.1687 & 0.4926 & $(--)$ & $\hat{a}_{\mathrm{p}}>\hat{a}_{\mathrm{M}}>\hat{a}_{\mathrm{w}}$ \\
\hline & 5 & 156 & $\begin{array}{l}{[110.0 ;} \\
\text { [IGF1; }\end{array}$ & $\begin{array}{r}157.9] \\
\text { SW967] }\end{array}$ & 3.76 & 0.6173 & 0.6432 & $<0.0001$ & (mat) & $\hat{a}_{\mathrm{M}}=\hat{\mathrm{a}}_{\mathrm{p}}=\hat{\mathrm{a}}_{\mathrm{w}}$ \\
\hline & 6 & 85 & $\begin{array}{l}\text { [73.7; } \\
\text { [FTO; }\end{array}$ & $\begin{array}{r}94.4] \\
\text { ETH5001] }\end{array}$ & 3.43 & 0.0036 & 0.0573 & 0.0700 & $(--)$ & $\hat{a}_{\mathrm{M}}=\hat{a}_{\mathrm{p}}>\hat{\mathrm{a}}_{\mathrm{w}}$ \\
\hline & 7 & 63 & $\begin{array}{l}{[0.0 ;} \\
\text { [S0025; }\end{array}$ & $\begin{array}{r}73.3] \\
\text { CYPD] }\end{array}$ & 3.56 & $<0.0001$ & 0.2359 & 0.4437 & $(--)$ & $\hat{a}_{\mathrm{m}}>\hat{a}_{\mathrm{p}}>\hat{a}_{\mathrm{w}}$ \\
\hline & 8 & 12 & $\begin{array}{l}{[0.0 ;} \\
\text { [SW905; }\end{array}$ & $\begin{array}{r}34.0] \\
\text { SW933] }\end{array}$ & 5.18 & $<0.0001$ & 0.2696 & 0.0707 & $(--)$ & $\hat{a}_{p}>\hat{a}_{M}>\hat{a}_{w}$ \\
\hline \multirow[t]{7}{*}{$\mathrm{CL}$} & 1 & 110 & $\begin{array}{l}{[77.3 ;} \\
\text { [SW307; }\end{array}$ & $\begin{array}{r}119.2] \\
\text { S0082] }\end{array}$ & 3.73 & 0.0873 & 0.0248 & 0.0021 & (mat) & $\hat{a}_{\mathrm{m}}=\hat{\mathrm{a}}_{\mathrm{p}}>\hat{\mathrm{a}}_{\mathrm{w}}$ \\
\hline & 1 & 161 & $\begin{array}{l}\text { [149.6; } \\
\text { [TGFBR1; }\end{array}$ & $\begin{array}{r}178.5] \\
\text { SW705] }\end{array}$ & 9.26 & $<0.0001$ & 0.1989 & 0.2241 & $(--)$ & $\hat{a}_{p}>\hat{a}_{m}>\hat{a}_{w}$ \\
\hline & 3 & 58 & $\begin{array}{l}{[35.9 ;} \\
{[S 0206 ;}\end{array}$ & $\begin{array}{r}74.0] \\
\text { SW828] }\end{array}$ & 3.63 & 0.1496 & 0.0005 & 0.3775 & $(--)$ & $\hat{a}_{\mathrm{m}}=\hat{a}_{\mathrm{p}}=\hat{a}_{\mathrm{w}}$ \\
\hline & 4 & 73 & $\begin{array}{l}{[62.1 ;} \\
{[S W 1073 ;}\end{array}$ & $\begin{array}{r}81.0] \\
\text { CASQ1] }\end{array}$ & 9.45 & $<0.0001$ & 0.0053 & 0.0424 & (nc) & $\hat{a}_{p}>\hat{a}_{M}>\hat{a}_{w}$ \\
\hline & 7 & 73 & $\begin{array}{l}\text { [61.3; } \\
\text { [ID4_ECO; }\end{array}$ & $\begin{array}{r}75.2] \\
; \quad \mathrm{KE} 6]\end{array}$ & 15.32 & $<0.0001$ & 0.1573 & 0.2116 & $(--)$ & $\hat{a}_{\mathrm{m}}=\hat{a}_{\mathrm{p}}>\hat{a}_{\mathrm{w}}$ \\
\hline & 8 & 13 & $\begin{array}{l}{[0.0 ;} \\
{[S W 905 ;}\end{array}$ & $\begin{array}{r}34.0] \\
\text { SW933] }\end{array}$ & 3.89 & $<0.0001$ & 0.4477 & 0.2922 & $(--)$ & $\hat{a}_{m}=\hat{a}_{p}>\hat{a}_{w}$ \\
\hline & 10 & 65 & $\begin{array}{l}{[52.5 ;} \\
\text { [SW497; }\end{array}$ & $\begin{array}{r}74.1] \\
\text { GAS1] }\end{array}$ & 3.03 & 0.1906 & 0.1264 & 0.0083 & (mat) & $\hat{a}_{\mathrm{m}}=\hat{a}_{\mathrm{p}}=\hat{a}_{\mathrm{w}}$ \\
\hline \multirow[t]{3}{*}{ FCR } & 1 & 105 & $\begin{array}{l}{[77.3 ;} \\
\text { [SW2130; }\end{array}$ & $\begin{array}{r}119.2] \\
\text { S0082] }\end{array}$ & 4.23 & 0.0856 & 0.0018 & 0.0105 & (mat) & $\hat{a}_{\mathrm{m}}>\hat{a}_{\mathrm{p}}=\hat{a}_{\mathrm{w}}$ \\
\hline & 3 & 41 & $\begin{array}{l}{[11.6 ;} \\
\text { [SW72; }\end{array}$ & $\begin{array}{r}74.0] \\
\text { SW828] }\end{array}$ & 3.46 & 0.0021 & 0.0605 & 0.7272 & $(--)$ & $\hat{a}_{\mathrm{m}}>\hat{a}_{\mathrm{p}}>\hat{a}_{\mathrm{w}}$ \\
\hline & 6 & 99 & {$[80.0$} & 102.4] & 3.25 & 0.0003 & 0.1463 & 0.9540 & $(--)$ & $\hat{a}_{\mathrm{m}}>\hat{a}_{\mathrm{p}}=\hat{a}_{\mathrm{w}}$ \\
\hline
\end{tabular}

$\mathrm{Cl}$ : confidence interval, $\mathrm{P}_{\text {add }}$ : error probability for additive effects, $\mathrm{P}_{\text {dom }}$ : error probability for dominant effects, $\mathrm{P}_{\text {imp }}$ : error probability for imprinting effects, Mode: mode of imprinting; (--) imprinting not significant, (mat) maternal imprinting, (pat) paternal imprinting, (nc) not consistent), $\hat{a}_{\mathrm{p}}$ : estimated effect of Pietrain breed, $\hat{a}_{\mathrm{m}}$ : estimated effect of Meishan breed, $\hat{a}_{\mathrm{w}}$ : estimated effect of Wild Boar breed 
Table 5

QTL results for muscling traits

\begin{tabular}{|c|c|c|c|c|c|c|c|c|c|c|}
\hline \multirow{2}{*}{$\frac{\text { Trait }}{\text { HMW }}$} & \multirow{2}{*}{$\frac{\text { SSC }}{1}$} & \multirow{2}{*}{$\frac{\text { Pos }}{66}$} & \multicolumn{2}{|l|}{$\mathrm{Cl}$} & \multirow{2}{*}{$\frac{\text { F-value }}{5.81}$} & \multirow{2}{*}{$\frac{P_{\text {add }}}{<0.0001}$} & \multirow{2}{*}{$\frac{P_{\text {dom }}}{0.9619}$} & \multirow{2}{*}{$\frac{P_{\text {imp }}}{0.0899}$} & \multirow{2}{*}{$\frac{\text { Mode }}{(--)}$} & \multirow{2}{*}{$\frac{\text { Order of effects }}{\hat{a}_{p}>\hat{a}_{M}>\hat{a}_{w}}$} \\
\hline & & & $\begin{array}{l}\text { [43.5; } \\
\text { [SWR2300: }\end{array}$ & $\begin{array}{r}77.3] \\
\text {.SW2130 }\end{array}$ & & & & & & \\
\hline & 1 & 119 & {$[110.3 ;$} & 126.3] & 3.36 & 0.0004 & 0.1097 & 0.2797 & $(--)$ & $\hat{a}_{p}>\hat{a}_{M}>\hat{a}_{w}$ \\
\hline & & & [SW307; & SW780 & & & & & & \\
\hline & 2 & 34 & $\begin{array}{l}\text { [14.9; } \\
\text { [SW2623; }\end{array}$ & $\begin{array}{r}68.0] \\
\text { MLP }\end{array}$ & 4.23 & 0.0080 & 0.7878 & 0.0006 & (mat) & $\hat{a}_{M}=\hat{a}_{p}>\hat{a}_{w}$ \\
\hline & 3 & 0 & $\begin{array}{l}\text { [0.0; } \\
\text { [SERPINE1; }\end{array}$ & $\begin{array}{r}11.6] \\
\text { SW72 }\end{array}$ & 3.94 & $<0.0001$ & 0.6002 & 0.3853 & $(--)$ & $\hat{a}_{M}=\hat{a}_{p}>\hat{a}_{w}$ \\
\hline & 4 & 71 & $\begin{array}{l}{[62.1 ;} \\
{[S W 1073 ;}\end{array}$ & $\begin{array}{r}75.3] \\
S 0073\end{array}$ & 6.95 & $<0.0001$ & 0.2454 & 0.2363 & $(--)$ & $\hat{a}_{\mathrm{p}}>\hat{a}_{\mathrm{m}}>\hat{a}_{\mathrm{w}}$ \\
\hline & 5 & 120 & $\begin{array}{l}{[110.0 ;} \\
\text { [IGF1; }\end{array}$ & $\begin{array}{l}150.4] \\
\text { MYF5 }\end{array}$ & 5.18 & 0.0002 & 0.9961 & $<0.0001$ & (mat) & $\hat{a}_{M}=\hat{a}_{p}>\hat{a}_{w}$ \\
\hline & 6 & 98 & $\begin{array}{l}\text { [80.0; } \\
\text { [S0087; }\end{array}$ & $\begin{array}{r}106.0] \\
\text { SKI }\end{array}$ & 5.76 & $<0.0001$ & 0.3880 & 0.2407 & $(--)$ & $\hat{a}_{p}>\hat{a}_{m}>\hat{a}_{w}$ \\
\hline & 7 & 73 & $\begin{array}{l}\text { [61.3; } \\
\text { [ID4_ECO; }\end{array}$ & $\begin{array}{r}86.5] \\
\text { S0102 }\end{array}$ & 4.06 & $<0.0001$ & 0.5281 & 0.4518 & $(--)$ & $\hat{a}_{M}>\hat{a}_{p}>\hat{a}_{w}$ \\
\hline & 8 & 15 & $\begin{array}{l}{[0.0 ;} \\
{[S W 905 ;}\end{array}$ & $\begin{array}{r}34.0] \\
\text { SW933 }\end{array}$ & 5.49 & $<0.0001$ & 0.4905 & 0.3259 & $(--)$ & $\hat{a}_{\mathrm{p}}>\hat{a}_{\mathrm{m}}>\hat{a}_{\mathrm{w}}$ \\
\hline & 10 & 63 & $\begin{array}{l}{[52.5 ;} \\
\text { [SW497; }\end{array}$ & $\begin{array}{r}\text { 74.1] } \\
\text { GAS1 }\end{array}$ & 5.03 & 0.0723 & 0.0052 & 0.0003 & (mat) & $\hat{a}_{p}>\hat{a}_{M}=\hat{a}_{w}$ \\
\hline & 12 & 95 & $\begin{array}{l}{[51.0 ;} \\
\text { [S0083; }\end{array}$ & $\begin{array}{l}109.8] \\
\text { S0106 }\end{array}$ & 2.85 & 0.0037 & 0.3635 & 0.0770 & $(--)$ & $\hat{a}_{p}>\hat{a}_{m}=\hat{a}_{w}$ \\
\hline & 14 & 91 & $\begin{array}{l}\text { [78.0; } \\
\text { [ACTA1; }\end{array}$ & $\begin{array}{r}105.1] \\
\text { SW2488 }\end{array}$ & 6.12 & 0.0001 & 0.5692 & 0.6072 & $(--)$ & $\hat{a}_{p}>\hat{a}_{m}>\hat{a}_{w}$ \\
\hline SMW & 1 & 119 & $\begin{array}{l}\text { [110.3; } \\
\text { [SW307; }\end{array}$ & $\begin{array}{r}126.3] \\
\text { SW780 }\end{array}$ & 5.39 & $<0.0001$ & 0.1457 & 0.0350 & (pat) & $\hat{a}_{p}>\hat{a}_{m}>\hat{a}_{w}$ \\
\hline & 2 & 48 & $\begin{array}{l}{[0.0 ;} \\
{[S W 2443 ;}\end{array}$ & $\begin{array}{r}\text { 77.8] } \\
\text { UBL5 }\end{array}$ & 4.44 & 0.0001 & 0.5542 & 0.0107 & (nc) & $\hat{a}_{\mathrm{M}}=\hat{\mathrm{a}}_{\mathrm{p}}>\hat{\mathrm{a}}_{\mathrm{w}}$ \\
\hline & 3 & 0 & $\begin{array}{l}{[0.0 ;} \\
\text { [SERPINE1; }\end{array}$ & $\begin{array}{r}11.6] \\
\text { SW72 }\end{array}$ & 4.73 & $<0.0001$ & 0.1081 & 0.6617 & $(--)$ & $\hat{a}_{\mathrm{M}}=\hat{a}_{\mathrm{p}}>\hat{\mathrm{a}}_{\mathrm{w}}$ \\
\hline & 3 & 56 & $\begin{array}{l}{[35.9 ;} \\
{[S 0206 ;}\end{array}$ & $\begin{array}{r}74.0] \\
\text { SW828 }\end{array}$ & 3.40 & 0.0993 & 0.0007 & 0.5296 & $(--)$ & $\hat{a}_{p}>\hat{a}_{m}=\hat{a}_{w}$ \\
\hline & 4 & 68 & $\begin{array}{l}{[62.1 ;} \\
{[S W 1073 ;}\end{array}$ & $\begin{array}{r}75.3] \\
\text { S0073 }\end{array}$ & 8.98 & $<0.0001$ & 0.4658 & 0.2340 & $(--)$ & $\hat{a}_{p}>\hat{a}_{M}>\hat{a}_{w}$ \\
\hline & 5 & 120 & $\begin{array}{l}{[77.3 ;} \\
\text { [S0005; }\end{array}$ & $\begin{array}{l}150.4] \\
\text { MYF5 }\end{array}$ & 3.69 & 0.0043 & 0.9294 & 0.0011 & (mat) & $\hat{a}_{\mathrm{p}}>\hat{a}_{\mathrm{M}}=\hat{a}_{\mathrm{w}}$ \\
\hline & 6 & 72 & $\begin{array}{l}{[58.1 ;} \\
\text { [SW1057; }\end{array}$ & $\begin{array}{r}80.0] \\
\text { S0087 }\end{array}$ & 3.90 & 0.0024 & 0.1828 & 0.0106 & (mat) & $\hat{a}_{p}>\hat{a}_{M}>\hat{a}_{w}$ \\
\hline & 7 & 70 & $\begin{array}{l}\text { [61.3; } \\
\text { [ID4_ECO; }\end{array}$ & $\begin{array}{r}86.5] \\
\text { S0102 }\end{array}$ & 6.98 & $<0.0001$ & 0.5094 & 0.1476 & $(--)$ & $\hat{a}_{M}>\hat{a}_{p}>\hat{a}_{w}$ \\
\hline & 8 & 12 & $\begin{array}{l}{[0.0 ;} \\
{[S W 905 ;}\end{array}$ & $\begin{array}{r}34.0] \\
\text { SW933 }\end{array}$ & 5.67 & $<0.0001$ & 0.3958 & 0.6961 & $(--)$ & $\hat{a}_{\mathrm{p}}>\hat{a}_{\mathrm{m}}>\hat{a}_{\mathrm{w}}$ \\
\hline & 10 & 65 & $\begin{array}{l}{[30.6 ;} \\
\text { [SW443; }\end{array}$ & $\begin{array}{r}\text { 74.1] } \\
\text { GAS1 }\end{array}$ & 3.75 & 0.2838 & 0.1446 & 0.0004 & (mat) & $\hat{a}_{M}=\hat{a}_{p}>\hat{a}_{w}$ \\
\hline LNMW & 1 & 66 & $\begin{array}{l}\text { [43.5; } \\
\text { [SWR2300; }\end{array}$ & $\begin{array}{r}77.3] \\
\text {; SW2130 }\end{array}$ & 7.10 & $<0.0001$ & 0.9358 & 0.1127 & $(--)$ & $\hat{a}_{\mathrm{p}}>\hat{a}_{\mathrm{m}}>\hat{a}_{\mathrm{w}}$ \\
\hline & 1 & 119 & $\begin{array}{l}{[110.3 ;} \\
\text { [SW307; }\end{array}$ & $\begin{array}{r}126.3] \\
\text { SW780 }\end{array}$ & 1.93 & 0.0336 & 0.1941 & 0.5139 & $(--)$ & $\hat{a}_{p}>\hat{a}_{M}=\hat{a}_{w}$ \\
\hline & 1 & 162 & $\begin{array}{l}{[149.6 ;} \\
\text { [TGFBR1; }\end{array}$ & $\begin{array}{r}178.5] \\
\text { SW705 }\end{array}$ & 3.28 & 0.0121 & 0.0133 & 0.1105 & $(--)$ & $\hat{a}_{\mathrm{p}}>\hat{a}_{\mathrm{m}}>\hat{a}_{\mathrm{w}}$ \\
\hline & 2 & 25 & $\begin{array}{l}{[5.2 ;} \\
{[S W C 9 ;}\end{array}$ & $\begin{array}{r}52.9] \\
\text { SW240 }\end{array}$ & 3.36 & 0.0033 & 0.1639 & 0.1554 & $(--)$ & $\hat{a}_{p}>\hat{a}_{M}>\hat{a}_{w}$ \\
\hline & 3 & 55 & $\begin{array}{l}{[35.9 ;} \\
\text { [S0206; }\end{array}$ & $\begin{array}{r}74.0] \\
\text { SW828 }\end{array}$ & 4.65 & 0.0003 & 0.0006 & 0.9149 & $(--)$ & $\hat{a}_{p}>\hat{a}_{M}>\hat{a}_{w}$ \\
\hline & 4 & 71 & $\begin{array}{l}{[50.9 ;} \\
\text { [SW2128; }\end{array}$ & $\begin{array}{r}75.3] \\
\text { S0073 }\end{array}$ & 7.33 & $<0.0001$ & 0.2312 & 0.2368 & $(--)$ & $\hat{a}_{p}>\hat{a}_{M}>\hat{a}_{w}$ \\
\hline
\end{tabular}


Table 5 continued

QTL results for muscling traits

\begin{tabular}{|c|c|c|c|c|c|c|c|c|c|c|}
\hline Trait & \multirow{2}{*}{$\begin{array}{c}\text { SSC } \\
5\end{array}$} & \multirow{2}{*}{$\begin{array}{l}\text { Pos } \\
118\end{array}$} & \multicolumn{2}{|c|}{$\mathrm{Cl}$} & \multirow{2}{*}{$\frac{\text { F-value }}{3.69}$} & \multirow{2}{*}{$\frac{P_{\text {add }}}{0.0046}$} & \multirow{2}{*}{$\frac{P_{\text {dom }}}{0.8156}$} & \multirow{2}{*}{$\frac{P_{\text {imp }}}{0.0011}$} & \multirow{2}{*}{$\frac{\text { Mode }}{\text { (nc) }}$} & \multirow{2}{*}{$\begin{array}{c}\text { Order of effects } \\
\hat{a}_{w}>\hat{a}_{M}=\hat{a}_{p}\end{array}$} \\
\hline & & & [92.3; & 150.4] & & & & & & \\
\hline & 6 & 88 & {$[80.0$} & $\begin{array}{l}\text { IVIrJ } \\
99.5]\end{array}$ & 4.91 & $<0.0001$ & 0.0385 & 0.2132 & $(--)$ & $\hat{a}_{\mathrm{s}}>\hat{a}_{\mathrm{m}}>\hat{a}_{\mathrm{w}}$ \\
\hline & & & [S0087; & TGFB1 & & & & & & \\
\hline & 8 & 13 & {$[0.0 ;$} & 34.0] & 4.89 & $<0.0001$ & 0.2773 & 0.1846 & $(--)$ & $\hat{a}_{p}>\hat{a}_{M}>\hat{a}_{w}$ \\
\hline & & & [SW905; & SW933 & & & & & & \\
\hline & 10 & 61 & {$[30.6 ;$} & 74.1] & 3.97 & 0.6031 & 0.0090 & 0.0006 & (nc) & $\hat{a}_{\mathrm{m}}=\hat{a}_{\mathrm{p}}=\hat{a}_{\mathrm{w}}$ \\
\hline & & & [SW443; & GAS1 & & & & & & \\
\hline & 14 & 65 & {$[43.8 ;$} & 105.1] & 3.70 & 0.0070 & 0.0031 & 0.2850 & $(--)$ & $\hat{a}_{p}>\hat{a}_{M}>\hat{a}_{w}$ \\
\hline & & & [SW2038; & SW2515 & & & & & & \\
\hline \multirow[t]{14}{*}{ MA } & 1 & 160 & {$[144.7 ;$} & 178.5] & 5.74 & $<0.0001$ & 0.4987 & 0.0042 & (pat) & $\hat{a}_{p}>\hat{a}_{w}>\hat{a}_{M}$ \\
\hline & & & [TPM2; & SW705 & & & & & & \\
\hline & 2 & 4 & {$[0.0$} & 14.9] & 4.72 & 0.0049 & 0.0019 & 0.0053 & (mat) & $\hat{a}_{\mathrm{m}}=\hat{a}_{\mathrm{p}}>\hat{a}_{\mathrm{w}}$ \\
\hline & & & [SW2443; & SW2623 & & & & & & \\
\hline & 4 & 71 & {$[62.1 ;$} & 75.3] & 4.27 & $<0.0001$ & 0.9746 & 0.6095 & $(--)$ & $\hat{a}_{p}>\hat{a}_{m}>\hat{a}_{w}$ \\
\hline & & & [SW1073; & S0073 & & & & & & \\
\hline & 6 & 94 & {$[80.0 ;$} & 99.5] & 4.65 & $<0.0001$ & 0.0444 & 0.2910 & $(--)$ & $\hat{a}_{p}>\hat{a}_{m}>\hat{a}_{w}$ \\
\hline & & & [S0087; & TGFB1 & & & & & & \\
\hline & 8 & 23 & {$[0.0$} & 49.4] & 4.31 & $<0.0001$ & 0.2301 & 0.1895 & $(--)$ & $\hat{a}_{p}>\hat{a}_{M}=\hat{a}_{w}$ \\
\hline & & & [SW905; & SW1070 & & & & & & \\
\hline & 8 & 96 & {$[49.4 ;$} & 110.1] & 3.04 & 0.0164 & 0.0095 & 0.6006 & $(--)$ & $\hat{a}_{p}>\hat{a}_{M}=\hat{a}_{w}$ \\
\hline & & & [SW1070; & SW16 & & & & & & \\
\hline & 14 & 77 & {$[60.7 ;$} & 105.1] & 5.69 & $<0.0001$ & 0.5742 & 0.5435 & $(--)$ & $\hat{a}_{p}>\hat{a}_{m}>\hat{a}_{w}$ \\
\hline & & & [SW540; & SW2488 & & & & & & \\
\hline
\end{tabular}

$\mathrm{Cl}$ : confidence interval, $\mathrm{P}_{\text {add }}$ : error probability for additive effects, $\mathrm{P}_{\mathrm{dog}}$ : error probability for dominant effects, $\mathrm{P}_{\text {imp }}$ : error probability for imprinting effects, Mode: mode of imprinting; (--) imprinting not significant, (mat) maternal imprinting, (pat) paternal imprinting, (nc) not consistent), $\hat{a}_{\mathrm{p}}$ : estimated effect of Pietrain breed, $\hat{a}_{\mathrm{m}}$ : estimated effect of Meishan breed, $\hat{a}_{\mathrm{w}}$ : estimated effect of Wild Boar breed

Most of the QTL were found on SSC1, 2, 3, 4, 6 and 8, and no QTL were on SSC9, 11, 13, 15, 17 and 18 (Figure 1). For the six growth traits, a total of 28 QTL were found, 12 with a significant dominant QTL effect and 10 with a significant imprinting QTL effect. For the four muscling traits, 40 QTL were found, with 10 and 12 significant dominant and imprinting effects, respectively. Most QTL were significant due to their additive effects. Some QTL, however, showed only a significant dominant and/or a significant imprinting effect, but no significant additive effects. Consequently, no different Mendelian alleles could be observed for these $\mathrm{QTL}$, and $\hat{a}_{p}=\hat{a}_{M}=\hat{a}_{w}$. For example, see QTL on SSC3 for CL and SMW, SSC5 for SW, SSC10 for HMW and SMW and SSC12 for W35. Many QTL showed similar position estimates and overlapping confidence intervals. The QTL with significant imprinting effects were mainly located on chromosomes 1, 2, 5 and 10. The mode of imprinting (paternal or maternal) was not always consistent across the three crosses. This can be interpreted as evidence against real imprinting effects, because it is not likely that an imprinted gene has a different mode in different crosses. As discussed in detail by Rückert \& Bennewitz (2010), the test for imprinting as conducted in this study might also reveal significance due to within founder breed segregation rather than due to real imprinting.

Due to the high number of mapped QTL not all of them will be discussed. A comparison of the results and other literature results can be done using the pig QTL data base (Hu et al. 2005). In the following, some interesting chromosomal regions will be considered and putative candidate genes underlying the QTL will be discussed. 


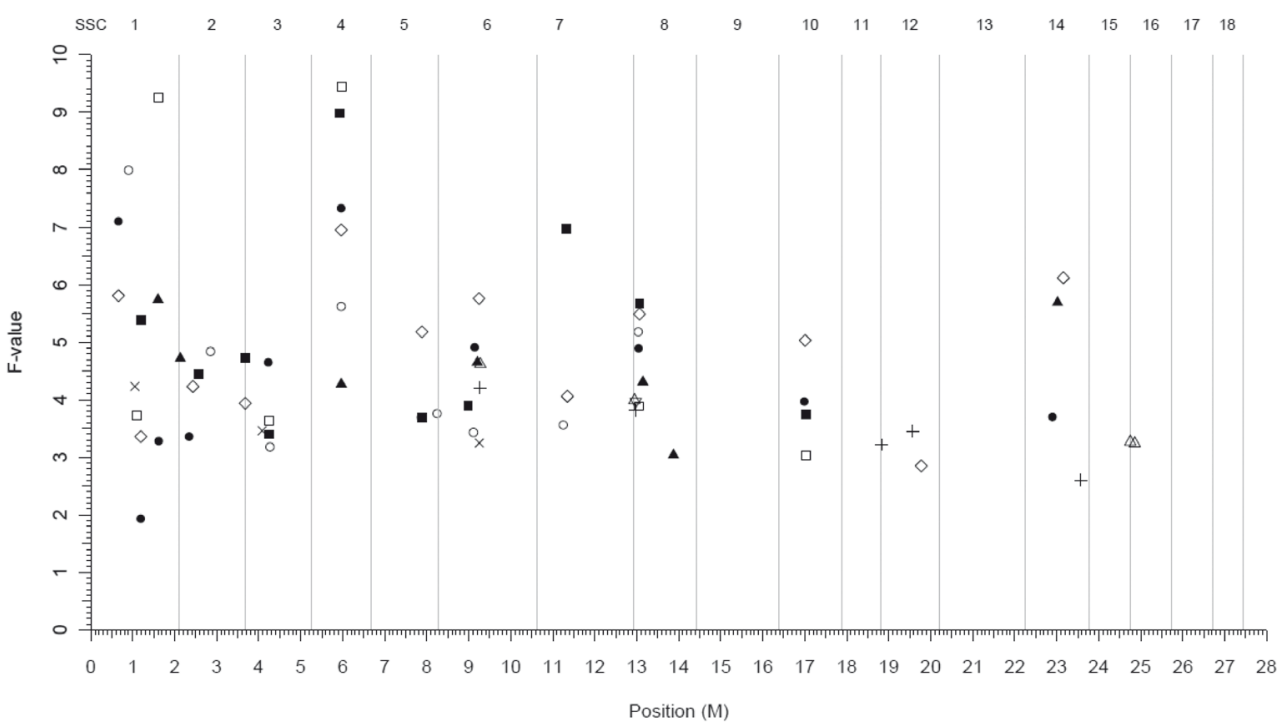

Figure 1

Overview of the QTL distribution of the porcine genome. Note that the test statistic of the QTL for CL on SSC7 was $\mathrm{F}>15$ (not shown in the figure). The definition of the symbols is given in Table 2.

For all traits except BW, W21, and W35 one or two QTL were found on SSC1. These QTL were distributed over five confidence intervals (see also the plots of the test statistics in Figure 2). QTL affecting growth and muscling on this chromosome have previously been mentioned in other $F_{2}$ cross-studies (Bidanel et al. 2001, Milan et al. 2002), although the QTL were not always located at the same region as in this study.

QTL were found for all muscling traits on SSC2. This is in agreement with Varona et al. (2002). A maternal imprinting effect was found for HMW and MA. The confidence intervals of these two QTL contain the IGF2 locus (co-localised with the microsatellite SWC9) which affects muscling and fattening traits and is known to be imprinted (Nezer et al. 1999). However, due to the large confidence intervals it might be that these imprinted QTL are caused by other imprinted genes, e.g. INS2 (Jeon et al. 1999). For SW a QTL was mapped in the interval between MYOD1 and InsR. Varona et al. (2002) also found significant QTL in this chromosomal region. MYOD1 is known to be involved in muscle differentiation and is mentioned as a candidate gene for growth (Fan et al. 2011).

QTL for some growth and muscling traits were found at the distal part of SSC3, with the SERPINE1 gene at the start of the confidence intervals. It codes for a protein called Serpine1, which is a molecule located in the extracellular space and is known to influence obesity and diabetes in humans (Kaur et al. 2010). SERPINE1 may be seen as possible candidate gene for growth. Additional QTL with a highly significant dominance effect were found for SW, CL, FCR, and LNMW.

The SSC4 is known as the chromosome with the highest density of QTL in pigs (Rothschild et al. 2007). In our study QTL were found for every trait, with a remarkably consistent chromosomal position estimated in the centromeric region (see also Figure 2). In this interval two markers located in the gene coding regions of VATP (coding for the vacuolar ATPase 

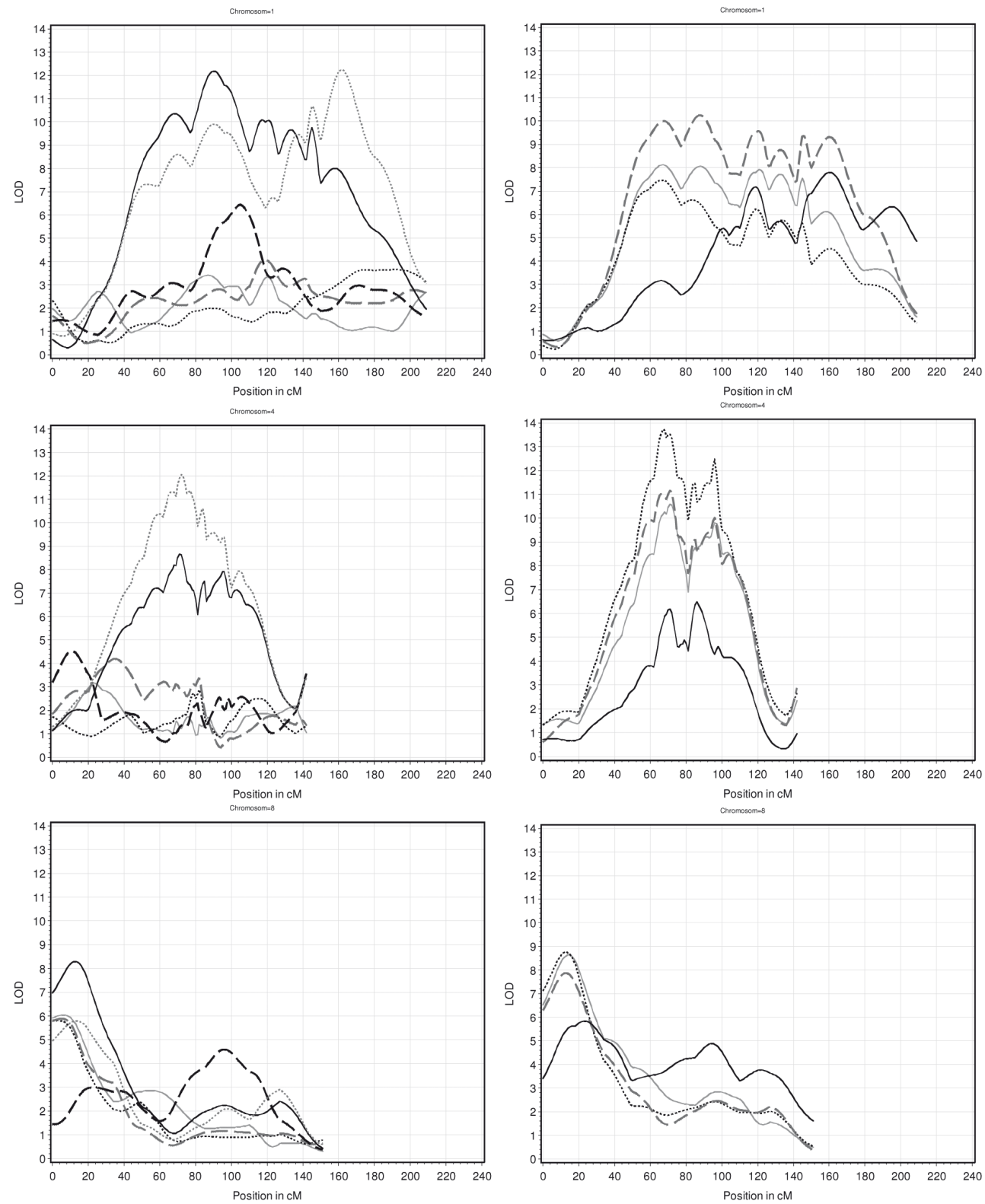

Figure 2

Plot of the test statistics for Chromosome 1 (top), 4 (middle) and 8 (bottom). Plots on the left show growth traits (BW: gray solid, W21: black dotted, W35: gray dashed, SW: black solid, CL: black dashed, FCR: gray dotted) and those on the right show muscling traits (HMW: gray solid, SMW: black dotted, LNMW: gray dashed, MA: black solid).

proton pump) and ATP1B1 (coding for the sodium/potassium-dependent ATPase beta-1 subunit) are of interest. Both gene products are involved in the ATP-dependent pathway including protein synthesis. 
Several QTL were found on SSC5 with highly significant imprinting effects and a consistent mode of imprinting, i.e. maternally imprinted. The confidence intervals included IGFI, which is known to be involved in a wide variety of growth responses (Fan et al. 2011) and has been suggested as a candidate gene (Roehe et al. 2003).

Porcine chromosome 6 is frequently mentioned in QTL studies, because several genes, such as the RYR1 (frequency of 0.492 and 0.513 in $\mathrm{W} \times \mathrm{P}$ and $\mathrm{M} \times \mathrm{P}$ in that study) associated with pale, soft and exudative meat and TGF- $\beta-1$, which controls cell growth, cell performance and cell differentiation, are located there. These two markers are within the overlapping QTL confidence intervals for six traits in our study. Additionally, Fan et al. (2009) detected a polymorphism within the fat mass and obesity associated protein gene (FTO), which is associated with growth and fatness traits. This gene is located at the bound of the QTL confidence intervals for SW and SMW in our study.

Many QTL have been detected in the same region of SSC7. Demars et al. (2007) searched for body composition traits on SSC7. In this study the same traits as in our study (slaughter weight, carcass length, ham and sholder weight) showed significant QTL in very similar regions of porcine chromosome 7. A QTL for carcass length on SSC7 was found by Sanchez et al. (2006). An exceptionally high test statistic (F-value 15) was found for a QTL for CL on SSC7 in this study. For this trait two other highly significant QTL (F-value >9) were also found. These high test statistic values were not observed for other traits. It seems that the low variation observed for $\mathrm{CL}$ is due to only a few genes with large effects. One possibly explanation might be that the genes affect the number of ribs. Therefore, candidate genes involved in determination of rib number were investigated. Two interesting candidate genes which are located close to the CL QTL on SSC7 were suggested. The first candidate gene is called PPARD, which is involved in cartilage development as well as in fat metabolism. Ren et al. (2011) described a missense mutation which is associated with ear size in Chinese pigs. The second one is the Bmp5 gene located at the short ear locus, which was investigated by Kingsley et al. (1992). Among others, Kingsley et al. (1992) demonstrated that null mutations at the Bmp5 locus reduce the number of ribs along the vertebral column. In further studies it should be investigated if the QTN in PPARD affects only the ear size or even CL (pleiotropy) or if the PPARD mutation is in LD with a mutation in the gene $B m p 5$. Therefore both candidate genes should be considered to unravel this exceptional QTL result.

Nine QTL were found on SSC8 (see Figure 2). In most cases the QTL were located in the distal region around the peroxisome proliferative activated receptor gamma coactivator 1 (PGCMUT or PPARGC1). PPARGC1 is a candidate gene that regulates the determination of myofibre types and has an important influence on myofibre growth (Jiang et al. 2011). In the study of Jiang et al. (2011), strong differences in gene expression between Landrace pigs and Chinese Meishans were reported. The detected QTL on SSC10 were all located in one region near the growth arrest-specific protein 1 marker (GAS1). GAS1 is an integral membrane protein and plays an important role in growth suppression in humans and mice (Del Sal et al. 1994).

The three QTL for muscling identified on SSC14 are located in the region around the marker actinin alpha 2 (ACTN2) and actin alpha 1 (ACTA1). Davoli et al. (2003) searched for polymorphisms in the myopalladine (MYOP) gene and placed the porcine MYOP gene, which is closely linked to ACTA1, on the genetic map of SSC14. Myopalladin (MYOP or FLJ14437) is 
a 145-kDa sarcomeric protein, which binds a-actinin with nebulin in skeletal muscle and functions in the organisation and assembly of the Z-line (Bang et al. 2001). Due to its role as a skeletal muscle gene especially coding for a sarcomeric protein, MYOP may play a key role in muscle mass accretion. Wimmers et al. (2007) searched for associations between functional candidate genes derived from gene-expression profiles of prenatal porcine muscle tissue and meat quality and muscle deposition. For MYOP the authors were able to show association with ham weight and lean content.

In conclusion, in this study the three connected $\mathrm{F}_{2}$-designs of Geldermann et al. (2003) were analysed jointly for muscling and growth traits using a multi-allele multi-QTL model. A large number of QTL was found compared to the separate analysis of crosses (see Geldermann et al. 2003). This underlines the high statistical power resulting from analysing the data jointly using an appropriate model. Based on small and overlapping confidence intervals, positional and functional candidate genes were suggested for most interesting QTL regions. In particular, the exceptional QTL for carcass length should be further investigated.

\section{References}

Andersson L, Haley CS, Ellegren H, Knott SA, Johansson M, Andersson K, Andersson-Eklund L, Edfors-Lilja I, Fredholm M, Hansson I, Håkansson J, Lundström K (1994) Genetic mapping of quantitative trait loci for growth and fatness in pigs. Science 263, 1771-1774

Bang ML, Mudry RE, McElhinny AS, Trombitás K, Geach AJ, Yamasaki R, Sorimachi H, Granzier H, Gregorio CC, Labeit S (2001) Myopalladin, a novel 145-kilodalton sarcomeric protein with multiple roles in Z-disc and I-band protein assemblies. J Cell Biol 153, 413-428

Bennewitz J, Reinsch N, Grohs C, Levéziel H, Malafosse A, Thomsen H, Xu N, Looft C, Kühn C, Brockmann GA, Schwerin M, Weimann C, Hiendleder S, Erhardt G, Medjugorac I, Russ I, Förster M, Brenig B, Reinhardt F, Reents R, Averdunk G, Blümel J, Boichard D, Kalm E (2003) Combined analysis of data from two granddaughter designs: A simple strategy for QTL confirmation and increasing experimental power in dairy cattle. Genet Sel Evol 35, 319-338

Bennewitz J, Meuwissen THE (2010) The distribution of QTL additive and dominance effects in porcine $F_{2}$ crosses. J Anim Breed Genet 127, 171-179

Bidanel JP, Milan D, lannuccelli N, Amigues Y, Boscher MY, Bourgeois F, Caritez JC, Gruand J, Le Roy P, Lagant H, Quintanilla R, Renard C, Gellin J, Ollivier L, Chevalet C (2001) Detection of quantitative trait loci for growth and fatness in pigs. Genet Sel Evol 33, 289-309

Davoli R, Braglia S, Lama B, Fontanesi L, Buttazzoni L, Baiocco C, Russo V (2003) Mapping, identification of polymorphisms and analysis of allele frequencies in the porcine skeletal muscle myopalladin and titin genes. Cytogenet Genome Res 102, 152-156

Del Sal G, Collavin L, Ruaro ME, Edomi P, Saccone S, Valle GD, Schneider C (1994) Structure, function, and chromosome mapping of the growth-suppressing human homologue of the murine gas 1 gene. Proc Natl Acad Sci 91, 1848-1852

Demars J, Riquet J, Sanchez MP, Billon Y, Hocquette JF, Lebret B, lannuccelli N, Bidanel JP, Milan D, Gondret F (2007) Metabolic and histochemical characteristics of fat and muscle tissues in homozygous or heterozygous pigs for the body composition QTL located an chromosome 7. Physiol Genomics 30, 232-241

Fan B, Du ZQ, Rothschild MF (2009) The fat mass and obesity-associated (FTO) gene is associated with intramuscular fat content and growth rate in the pig. Anim Biotechnol 20, 58-70

Fan B, Onteru SK, Du ZQ, Garrick DJ, Stalder KJ, Rothschild MF (2011) Genome-wide association study identifies loci for body composition and structural soundness traits in pigs. PLoS ONE 6, e14726. doi:10.1371/journal. pone. 0014726 
Geldermann H, Müller E, Moser G, Reiner G, Bartenschlager H, Cepica S, Stratil A, Kuryl J, Moran C, Davoli R, Brunsch C (2003) Genome-wide linkage and QTL mapping in porcine F2 families generated from Pietrain, Meishan and Wild Boar crosses. J Anim Breed Genet 120, 363-393

Hu ZL, Dracheva S, Jang W, Maglott D, Bastiaansen J, Rothschild MF, Reecy JM (2005) A QTL resource and comparison tool for pigs: PigQTLDB. Mammalian Genome 16, 792-800

Jeon JT, Carlborg Ö, Törnsten A, Giuffra E, Amarger V, Chardon P, Andersson-Eklund L, Andersson K, Hansson I, Lundström K, Andersson L (1999) A paternally expressed QTL affecting skeletal and cardiac muscle mass in pigs maps to the IGF2 locus. Nat Genet 21, 157-158

Jiang AA, Li MZ, Zhang K, Gu YR, Li XW (2011) Expression changes of PPARGC1A during the development of Lean and Obese pigs. J Anim Vet Adv 10, 597-600

Kaur P, Reis MD, Couchman GR, Forjuoh SN, Greene JF, Asea A (2010) SERPINE1 links obesity and diabetes: a pilot study. J Proteomics Bioinform 3, 191-199

Kingsley DM, Bland AE, Grubber JM, Marker PC, Russell LB, Copeland NG, Jenkins NA (1992) The mouse short ear skeletal morphogenesis locus is associated with defects in a bone morphogenetic member of the TGF $\beta$ superfamily. Cell 71, 399-410

Lynch M, Walsh B (1998) Genetics and Analysis of Quantitative Traits. Sunderland, Mass., USA

Milan D, Bidanel JP, lannuccelli N, Riquet J, Amigues Y, Gruand J, Le Roy P, Renard C, Chevalet C (2002) Detection of quantitative trait loci for carcass composition traits in pigs. Genet Sel Evol 34, 705-728

Nezer C, Moreau L, Brouwers B, Coppieters W, Detilleux J, Hanset R,. Karim L, Kvasz A, Leroy P and Georges M (1999) An imprinted QTL with major effect on muscle mass and fat deposition maps to the IGF2 locus in pigs. Nat Genet 21, 155-156

Piepho HP (2001) A quick method for computing approximate thresholds for quantitative trait loci detection. Genetics 157, 425-432

Reinsch N (1999) A multiple-species, multiple-project database for genotypes at codominant loci. J Anim Breed Genet 116, 425-435

Ren J, Duan Y, Qiao R, Yao F, Zhang Z, Yang B, Guo Y, Xiao S, Wei R, Ouyang Z, Ding N., Ai H, Huang L (2011) A Missense mutation in PPARD causes a major QTL effect on ear size in pigs. PLoS Genet 7, e1002043. doi:10.1371/journal.pgen.1002043

Rothschild MF, Hu ZL, Jiang Z (2007) Advances in QTL mapping in pigs. Int J Biol Sci 3, 192-197

Roehe R, Plastow GS, Knap PW (2003) Quantitative and molecular genetic determination of protein and fat deposition. Homo 54, 119-131

Rückert C, Bennewitz J (2010) Joint QTL analysis of three connected $F_{2}$-crosses on pigs. Genet Sel Evol 42, 40

Rückert C, Stratz P, Preuss S, Bennewitz J (2012) Mapping quantitative trait loci for metabolic and cytological fatness traits of connected F2 crosses in pigs. J Anim Sci 90, 399-409

Sanchez MP, Riquet J, lannuccelli N, Gogué J, Billon Y, Demeure O, Caritez JC, Burgaud G, Fève K, Bonnet M, Péry C, Lagant H, Le Roy P, Bidanel JP, Milan D (2006) Effects of quantitative trait loci on chromosomes 1 , 2,4 , and 7 on growth, carcass, and meat quality traits in backcross Meishan $\times$ Large White pigs. J Anim Sci 84, 526-537

Stratz P, Baes C, Rückert C, Preuss S, Bennewitz J. (2012) A two-step approach to map quantitative trait loci for meat quality in connected porcine $F_{2}$ crosses considering main and epistatic effects. Anim Genet (accepted)

Varona L, Ovilo C, Clop A, Noguera JL, Pérez-Enciso M, Coll A, Folch JM, Barragán C, Toro MA, Babot D, Sánchez A (2002) QTL mapping for growth and carcass traits in an Iberian by Landrace pig intercross: additive, dominant and epistatic effects. Genet Res 80, 145-154

Walling GA, Visscher PM, Andersson L, Rothschild MF, Wang L, Moser G, Groenen MAM, Bidanel JP, Cepica S, Archibald AL, Geldermann H, de Koning DJ, Milan D, Haley CS (2000) Combined analyses of data from quantitative trait loci mapping studies: Chromosome 4 effects on porcine growth and fatness. Genetics $155,1369-1378$ 
Wimmers K, Murani E, Te Pas MFW, Chang KC, Davoli R, Merks JWM, Henne H, Muraniova M, Da Costa N, Harlizius B, Schellander K, Böll I, Braglia S, De Wit AAC, Cagnazzo M, Fontanesi L, Prins D, Ponsuksili S (2007) Associations of functional candidate genes derived from gene-expression profiles of prenatal porcine muscle tissue with meat quality and muscle deposition. Anim Genet 38, 474-484

Received 1 March 2012, accepted 27 April 2012.

Corresponding author:

Jörn Bennewitz

email: j.bennewitz@uni-hohenheim.de

Institute of Animal Husbandry and Animal Breeding, Universität Hohenheim, Garbenstraße 17, 70599 Stuttgart-Hohenheim, Germany 